Baltzer Journals

April 3, 2018

\title{
Mobile ATM Buffer Capacity Analysis*
}

\author{
Stephen F. Bush ${ }^{\dagger}$, Joseph B. Evans AND Victor Frost \\ Telecommunications \& Information Sciences Laboratory \\ Department of Electrical Engineering \& Computer Science \\ University of Kansas \\ Lawrence, KS 66045-2228 \\ E-mail: sbush@tisl.ukans.edu
}

\begin{abstract}
This paper extends a stochastic theory for buffer fill distribution for multiple "on" and "off" sources to a mobile environment. Queue fill distribution is described by a set of differential equations assuming sources alternate asynchronously between exponentially distributed periods in "on" and "off" states. This paper includes the probabilities that mobile sources have links to a given queue. The sources represent mobile user nodes, and the queue represents the capacity of a switch. This paper presents a method of analysis which uses mobile parameters such as speed, call rates per unit area, cell area, and call duration and determines queue fill distribution at the ATM cell level. The analytic results are compared with simulation results.
\end{abstract}

\section{Introduction}

The next generation of mobile systems will most likely be B-ISDN compatible. This paper considers buffer fill distribution in a mobile ATM environment. The ATM protocol standard specifies fixed size 53 byte cells consisting of 48 bytes of payload and a 5 byte header. Because the ATM cell sizes are relatively small, there will be a moderate amount of cut-through. This means that there is an increase in performance because ATM cells will be available for use immediately after being de-encapsulated from the wireless media carrying the ATM cells. Also, mobile systems will be able to take advantage of the standardized QoS parameters as well as having an end-to-end standards based protocol with fixed networks. This will lead to standards based integration with fixed networks and integration of voice, data, and video.

$\mathrm{M} / \mathrm{D} / 1$ analysis of fixed size ATM cells provides optimistic results because M/D/1 assumes that sources are Poisson. A technique which does not make that assumption provides a more accurate analysis and is extended in this paper to a mobile environment.

It appears that there has been little work done concerning the effects of mobility on ATM. This paper will attempt to build a foundation for analyzing mobile ATM networks by extending previous work for fixed ATM environments. This analysis would be useful for determining the base station queue fill distribution and probability of cell loss in a mobile environment. It would also be useful for simplifying mobile CBR cell simulations.

${ }^{*}$ This paper is partially funded by ARPA contract number J-FBI-94-223.

${ }^{\dagger}$ The Mathematica code for this paper can be found on http://www.tisl.ukans.edu/ sbush. 


\section{Mobile Systems Analysis}

The equilibrium buffer fill distribution can be described by a set of differential equations assuming sources alternate asynchronously between exponentially distributed periods in "on" and "off" states. Figure 1 shows such a source. Note that the "on" to "off" probability is normalized to one and that the transitions represented are intensities.

\section{CBR Source}

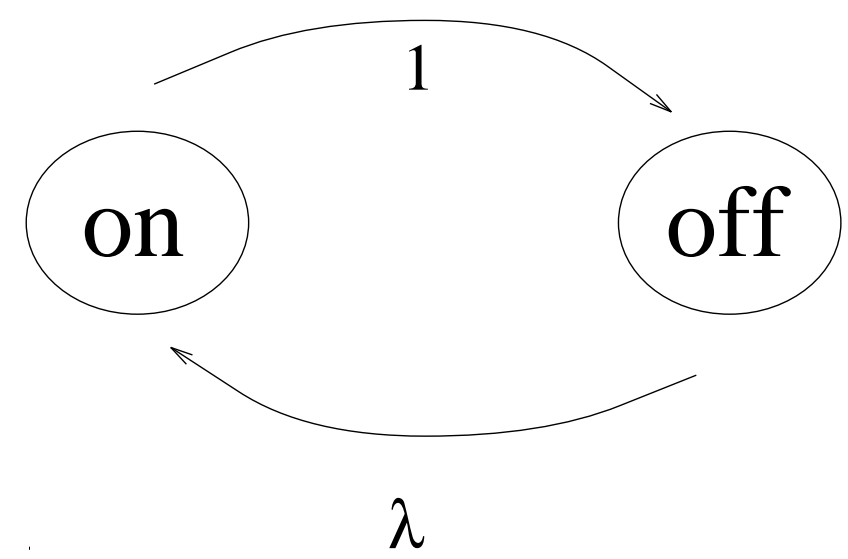

Figure 1: Constant Bit Rate “on”-“off” ATM Source.

In addition, the probabilities that mobile sources have links to a given buffer are included. The sources represent mobile user nodes which are transmitting and receiving ATM traffic, and the buffer represents a switch as shown in Figure 2. The details of such a mobile ATM system implementation are described in the Rapidly Deployable Radio Networks (RDRN) Network Architecture [3] and [2].

Figure 3 illustrates the handoff of a remote node from one switch to another. Note that the "on"-"off" CBR Source model is similar to the "connected"-“disconnected" status of the remote nodes as they handoff from one base station to another. This observation is used in developing the analysis for mobile ATM systems.

In a fixed network, the queue fill distribution is determined for multiple constant rate on-off sources. However, it assumes that the number of sources remains constant over a sufficient period of time for the equilibrium probabilities to be valid. There are at least two ways of extending the analysis to a mobile cellular environment.

Consider the ATM cell queue fill distribution at the base station. The simplest, but least accurate extension is to determine the average number of channels used per cell area as $t \rightarrow \infty$. However, there is nothing to stop mobile units from concentrating in a small number of cell areas at some time. There is a hard limit on the number of sources a base station will accept because each base station has a limited number of ports. Once this number is reached, further handoffs 


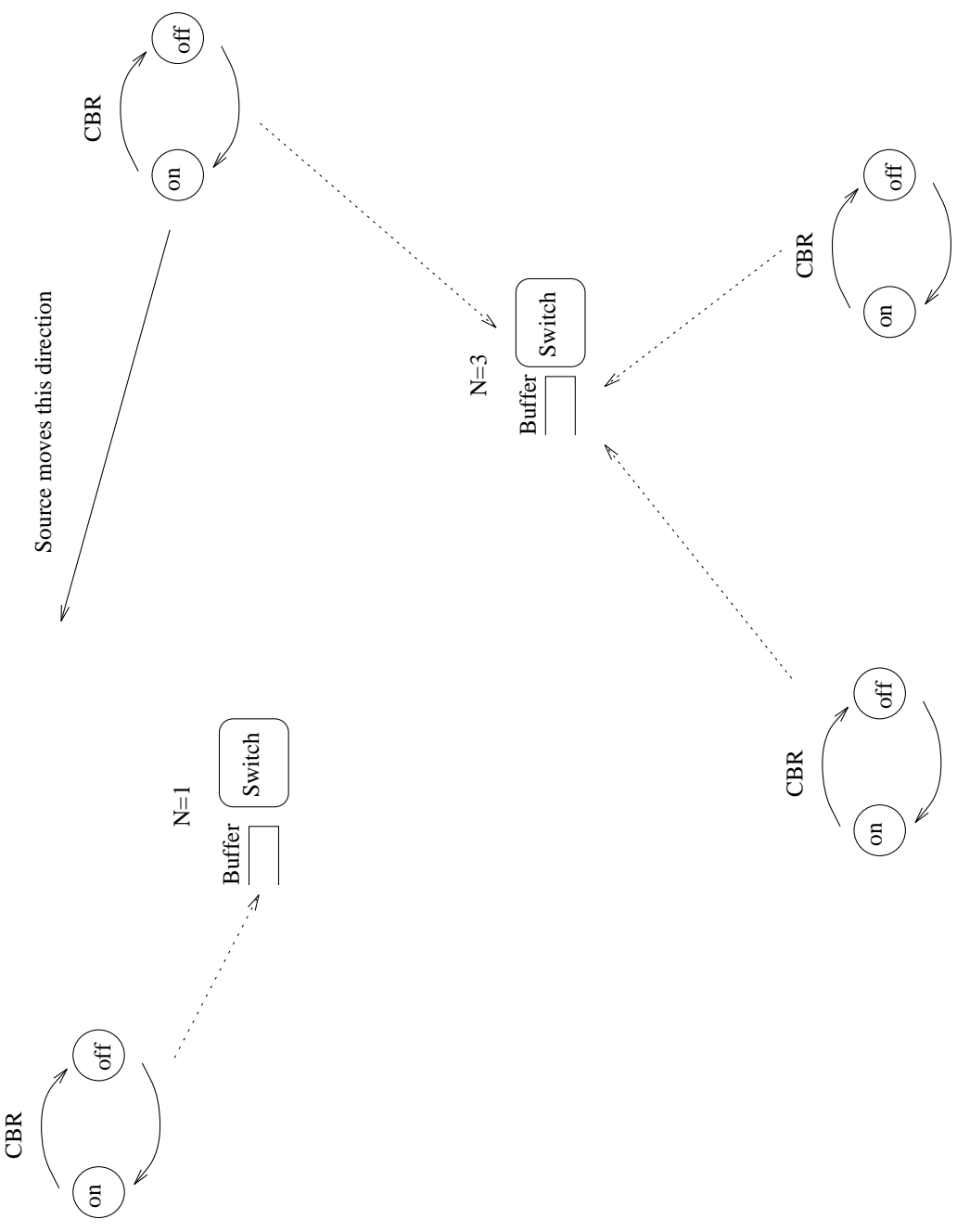

Figure 2: Mobile ATM Sources before Handoff. 
S. Bush, J. Evans and V. Frost / Mobile ATM Buffer Capacity Analysis
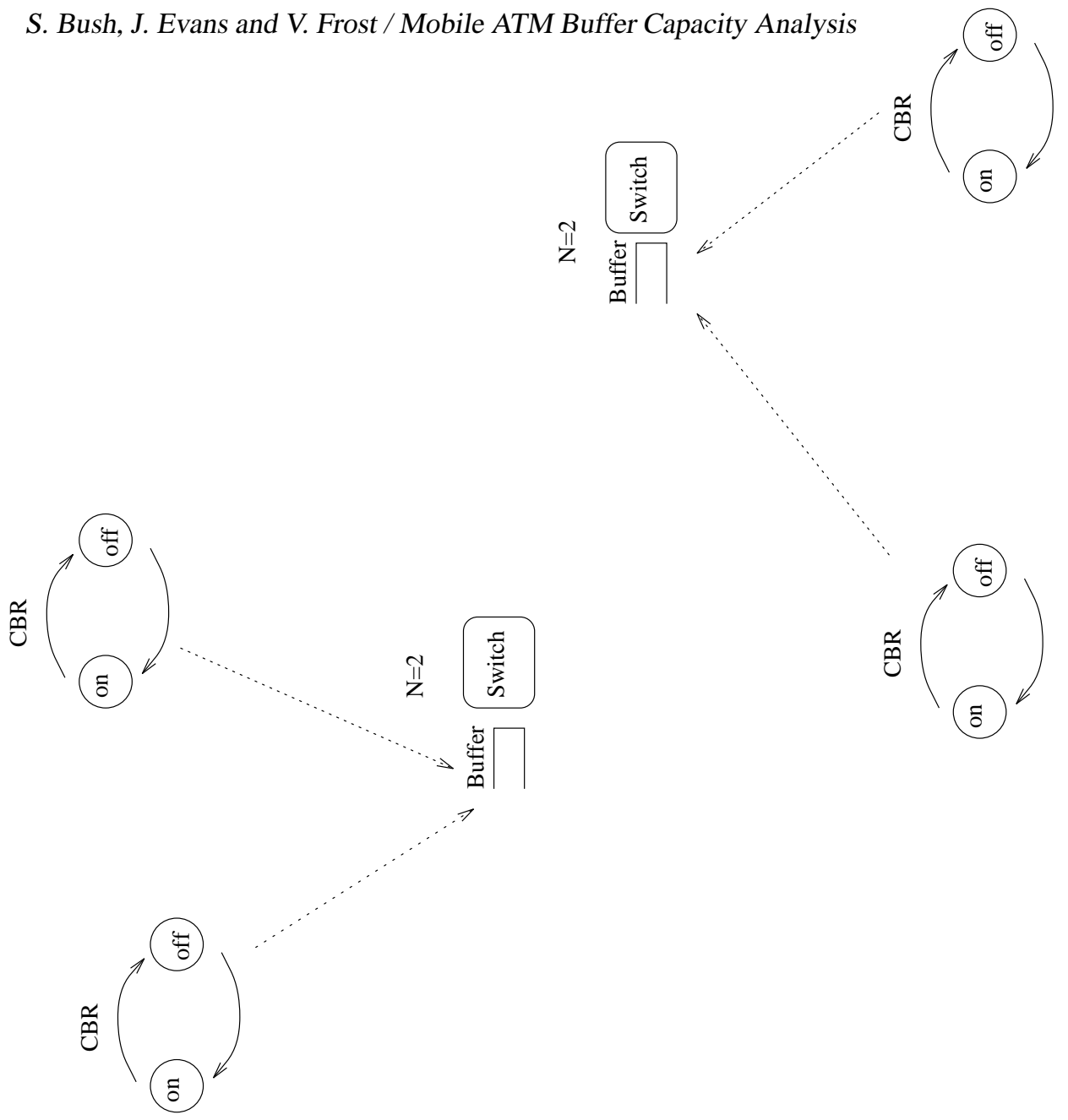

Figure 3: Mobile ATM Sources after Handoff. 
into such a cell will cause their connections to terminate. Thus, determining how many codes to assign to a base station is a critical design choice, [5].

Note that code assignment can be dynamic, but this will not be considered here in order to help simplify the analysis. Also, cell areas can be designed to overlap [5]. Although this can increase the probability of interference, it has a beneficial effect on handoff. When a mobile unit determines that a handoff is likely to occur and the cell it will enter has no channels available, the mobile unit can continue to use the cell within which it currently resides, and queue the handoff to the next cell. If a channel becomes available in the destination cell before the mobile leaves its current cell, the handoff can take place successfully. It would be interesting to see the effect of queuing the handoffs. Again, in order to keep the computation simple for this paper, this will not be considered.

This paper makes the simplifying assumption that each mobile unit is a single ATM source multiplexed at the base station. In general, each mobile unit would be a set of sources; however, this could again be a future extension of this paper.

\subsection{Mobile Node Analysis}

This paper makes use of the analysis and notation in [4]. There are two probability distributions that need to be developed: the channel holding time and the equilibrium probability of the number of channels used per base switch. The channel holding time is the probability that a particular base station's channel will be in use at a given time, or equivalently, that a particular source still exists. The equilibrium probability of channels in use for a given base switch is useful in this analysis as shown later.

The first of many simplifying assumptions is that there is a known average number of new calls per second per unit area. Let this be $\lambda_{R}$ where $R$ is the radius of the particular cell area. Handoffs are attempted at an average rate per cell, $\lambda_{R h}$. The ratio of handoff attempts to new call attempts will be $\gamma_{o} \triangleq \frac{\lambda_{R h}}{\lambda_{R}}$.

Let $P_{B}$ be the average number of new call attempts which are blocked. Then new calls are accepted at an average rate of $\lambda_{R c}=\lambda_{R}\left(1-P_{B}\right)$. Similarly, let $P_{f h}$ be the average number of handoff attempts which are blocked. Then handoff calls are accepted at a rate $\lambda_{R h c}=\lambda_{R h}(1-$ $\left.P_{f h}\right)$. The ratio of the average accepted handoffs to the average number of new calls accepted is

$\gamma_{c} \triangleq \frac{\lambda_{R h c}}{\lambda_{R c}}$. The channel holding time, $T_{h}$, is a random variable defined as the time beginning when a channel is accessed, either via a new call or handoff, until the channel is released, via handoff or call completion. In order to define this, another random variable, $T_{M}$ is defined. $T_{M}$ is the time duration of a call, regardless of handoff or blocking. It is simplified as an exponential with average value, $\frac{1}{\mu_{M}}$. Thus the pdf is

$$
f_{T_{M}}(t)=\mu_{M} e^{-\mu_{M} t}
$$

The strategy for determining the channel holding time distribution is to consider the time remaining for a call which has not been handed off yet, $T_{H n}$, and the time remaining after a handoff, $T_{H h}$. Since the call duration, $T_{M}$ is memoryless, the time remaining for a call after handoff has the same distribution as the original call duration. Let $T_{n}$ be the time the mobile unit remains in the original cell area, and $T_{h}$ be the time the mobile resides in the cell area after handoff. $T_{H n}$ is the minimum of the call duration, $T_{M}$, or the dwell time in the originating cell area, $T_{n}$. A similar reasoning applies to the cell area into which a mobile unit has moved after a handoff; $T_{H h}$ is the minimum of the call duration, $T_{M}$, or the dwell time in the cell area after 
handoff, $T_{h}$.

Thus,

$$
\begin{aligned}
& F_{T_{H n}}(t)=F_{T_{M}}(t)+F_{T_{n}}(t)\left(1-F_{T_{M}}(t)\right) \\
& F_{T_{H h}}(t)=F_{T_{M}}(t)+F_{T_{h}}(t)\left(1-F_{T_{M}}(t)\right)
\end{aligned}
$$

where $\left(1-F_{T_{M}}(t)\right)$ is the probability that a call does not complete within the current cell area.

The distribution of channel holding time in a particular cell area is a weighted function of the equations shown in 2 above,

$$
F_{T_{H}}(t)=\frac{\lambda_{R c}}{\lambda_{R c}+\lambda_{R h c}} F_{T_{H n}}(t)+\frac{\lambda_{R h c}}{\lambda_{R c}+\lambda_{R h c}} F_{T_{H h}}(t)
$$

Substituting the values from Equation 1,

$$
F_{T_{H}}(t)=1-e^{-\mu_{M} t}+\frac{e^{-\mu_{M} t}}{1+\gamma_{c}}\left(F_{T_{n}}(t)+\gamma_{c} F_{T_{h}}(t)\right)
$$

and differentiating to get the pdf,

$$
\begin{aligned}
f_{T_{H}}(t)= & \mu_{M} e^{-\mu_{M} t} \\
& +\frac{e^{-\mu_{M} t}}{1+\gamma_{c}}\left[f_{T_{n}}(t)+\gamma_{c} f_{T_{h}}(t)\right] \\
& -\frac{e^{-\mu_{M} t}}{1+\gamma_{c}}\left[F_{T_{n}}(t)+\gamma_{c} F_{T_{h}}(t)\right]
\end{aligned}
$$

To determine the equilibrium probability of the number of mobile hosts using a base station, approximate the channel holding time as simply an exponential distribution. The birth-death Markov chain can be used to find the equilibrium probability of the number of sources in each cell area. The up rates are $\lambda_{R}+\lambda_{R h}$ and the down rates are multiples of the mean channel holding time.

Putting the Markov chain in closed form,

$$
P_{j}=\frac{\left(\lambda_{R}+\lambda_{R h}\right)^{j}}{j ! \mu_{H}^{j}} P_{0}
$$

where,

$$
P_{0}=\frac{1}{\sum_{k=0}^{C} \frac{\left(\lambda_{R}+\lambda_{R} h\right)^{k}}{k ! \mu_{H}^{k}}}
$$

Note that $C$ is the total number of channels for a base station and handoffs will fail with probability $P_{C}$, i.e. all channels in that cell area are currently in use.

\subsection{Mobile CBR Source Analysis}

Assume that the number of mobile hosts in a cell area is independent of whether its CBR source is on or off. We can now modify the probability that $i$ sources are on and the queue fill is less than $x$ by incorporating the probability that there are at least $i$ sources, shown in Equation 8 .

$$
P_{i_{\text {mobile }}}(t, x) \triangleq P_{j \geq i} \text { and } P_{i}(t, x)
$$


$P_{i}(t, x)$ is the probability that at time $t, i$ sources are on, and the number of items in the buffer does not exceed $x . P_{j \geq i}$ is the probability that there are at least $i$ sources sending data to the buffer. $P_{j \geq i}$ can be found from Equation 6 as follows,

$$
P_{j \geq i}=\sum_{j=i}^{C} P_{j}
$$

The buffer fill distribution as defined in [1] is

$$
\begin{array}{r}
P_{i}(t+\Delta t, x)=\{N-(i-1)\} \lambda \Delta t P_{i-1}(t, x) \\
+(i+1) \Delta t P_{i+1}(t, x) \\
+\{1-((N-i) \lambda+i)\} \Delta t P_{i}(t, x-(i-c) \Delta t) \\
+O\left(\Delta t^{2}\right)
\end{array}
$$

Now that the channel equilibrium probabilities have been determined, we can account for the fact that the sources are mobile. Since the channel equilibrium probabilities have no dependence on time, the method of solution in [1] can be used with minor modifications,

$$
\begin{array}{r}
P_{i}(t+\Delta t, x)=\{N-(i-1)\} \lambda \Delta t P_{i-1}(t, x) P_{j \geq i-1} \\
+(i+1) \Delta t P_{i+1}(t, x) P_{j \geq i+1} \\
+\{1-((N-i) \lambda+i)\} \Delta t P_{i}(t, x-(i-c) \Delta t) P_{j \geq i} \\
+O\left(\Delta t^{2}\right)
\end{array}
$$

From [1], $F_{i}(x)$ is the equilibrium probability that $i$ sources are on, and the buffer content does not exceed $x$. Thus $F_{i}(\infty)$ is the probability that $i$ out of $N$ sources are simultaneously on. In the mobile environment, this is now,

$$
F_{i}(\infty)=\sum_{j=1}^{C} P_{j}\left(\begin{array}{l}
C \\
j
\end{array}\right)\left(\frac{\lambda}{1+\lambda}\right)^{j}\left(\frac{1}{1+\lambda}\right)^{C-j}
$$

The mobile extension from Equation 8 carries through [1] for example, equation (13) in [1] is now,

$$
\phi_{i_{\text {mobile }}} \triangleq \phi_{i} P_{j=i}
$$

and

$$
\Phi(1)=\sum_{i=0}^{C} \phi_{i} P_{j=i}
$$

$\phi_{i}$ is the right eigenvector of

$$
z D \phi=M \phi
$$

where $\mathrm{D}$ and $\mathrm{M}$ are matrices used to represent the differential equation in Equation 10.

$\Phi(x)$ is the generating function of $\phi$. These values are useful in [1] for solving the equilibrium buffer fill differential equation. The remainder of the solution is straight forward from [1]. Thus it has been shown how an analysis of constant bit rate on-off sources which model fixed length ATM packet sources, is extended to a mobile environment. 
Note that the analysis uses a technique which is more accurate than $M / D / 1$ for the fixed size ATM cells, yet uses a memoryless analysis for the channel holding time distribution. This is a reasonable approach since the variable length channel hold times can be accurately modeled by a memoryless analysis, while the $M / D / 1$ analysis yields optimistic results which can be replaced by the more accurate method in [1] as this section has described.

\subsection{Example}

The following is a simple example of the analysis using the same parameters as the simulation in the next section. The parameters required are:

- $\lambda_{R}=0.06 \mathrm{calls} / \mathrm{sec} / \mathrm{square}$ mile

- $T_{M}=40 \mathrm{secs}$

- $V_{\max }=0.03$ miles $/ \mathrm{sec}$

- $C=3$ channels per base station

From the equations in the previous section, we can develop an analytical solution for the remaining parameters. Using basic probability the integral of Equation 5 should be one. Also, $\mu_{H}$ is an exponential approximation of Equation 4, which can be found by taking the integral of the difference of $F_{T_{H}}(t)$ from Equation 4 and $\mu_{H}$ and setting the result to zero,

$$
\begin{gathered}
\int_{0}^{\infty} f_{T H}(t) d t=1 \\
\int_{0}^{\infty}\left[F_{T H}(t)-e^{\mu_{H} t}\right] d t=0 .
\end{gathered}
$$

This provides two equations and two unknowns which provide the solution for $\lambda_{R h}=2.16$ and $\mu_{H}=9.48$. These values can be used to determine the $P_{j}$ which can then be used in our extension of [1] as discussed previously. In the graph of $P_{j}$ shown in Figures 4, it appears that there will be a high probability of blocking, since $P_{B}=P_{C}=P_{3}$. This is compared with an arrival rate of 0.01 calls/sec/unit area in Figure 5, which has a maximum at one channel per station, and a lower blocking probability.

Figures 4 and 5 are in agreement with the simulation results in Figures 7 and 8 as additional verification.

\subsection{Simulation and Results}

The mobile communications system model ${ }^{1}$ is shown in figure 6 . It is an open system; mobile hosts are generated at rate with inter-arrival time specified by Exp Pulse Mean, initiate a call for an average exponential duration specified by Mean Session Length, and exit the system when either the call is complete or the mobile moves out of all cell areas.

The first step is to create the base stations and their cell areas. The total number of base stations is:

$$
\text { Base Station Matrix Size }{ }^{2}
$$

\footnotetext{
${ }^{1}$ A mobile cellular telephone system library comes with the BONeS software. As much as possible of that library is used as a basis for this simulation.
} 


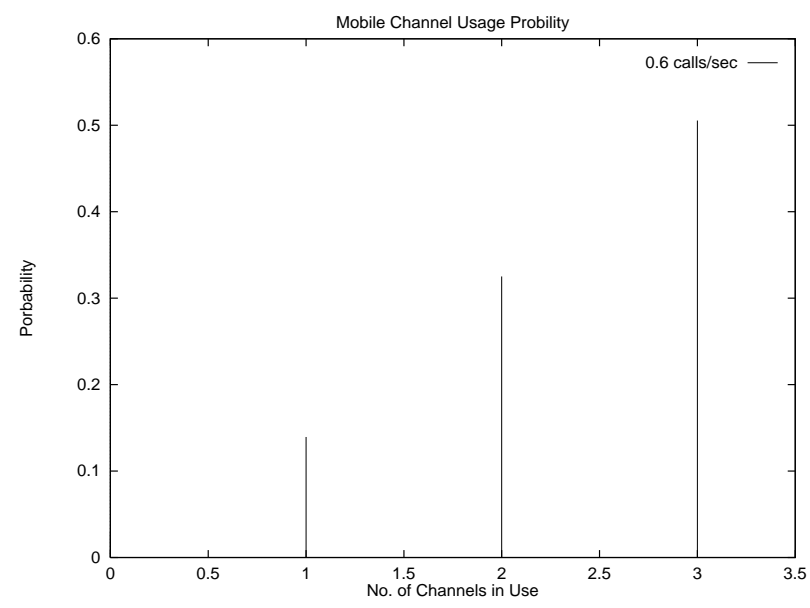

Figure 4: Channel Usage Prob. Density Function for 0.6 Call/Sec.

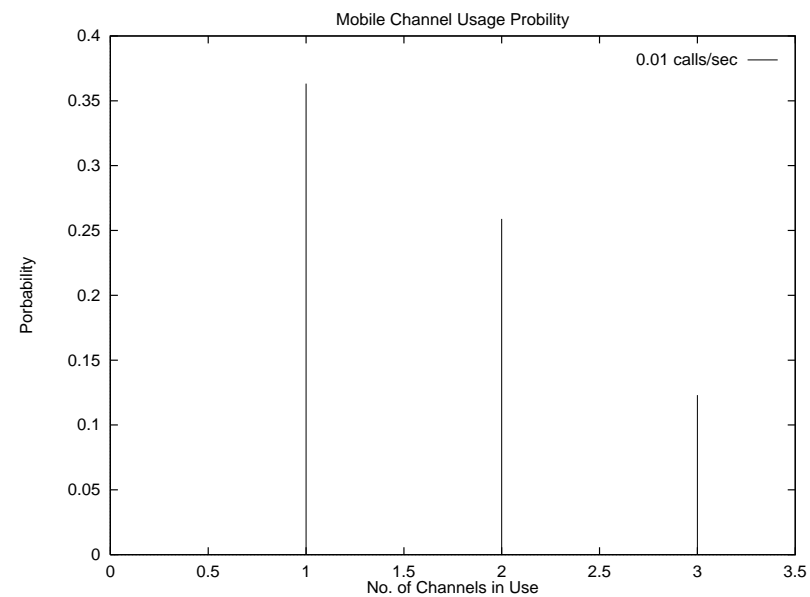

Figure 5: Channel Usage Prob. Density Function for 0.01 Call/Sec. 


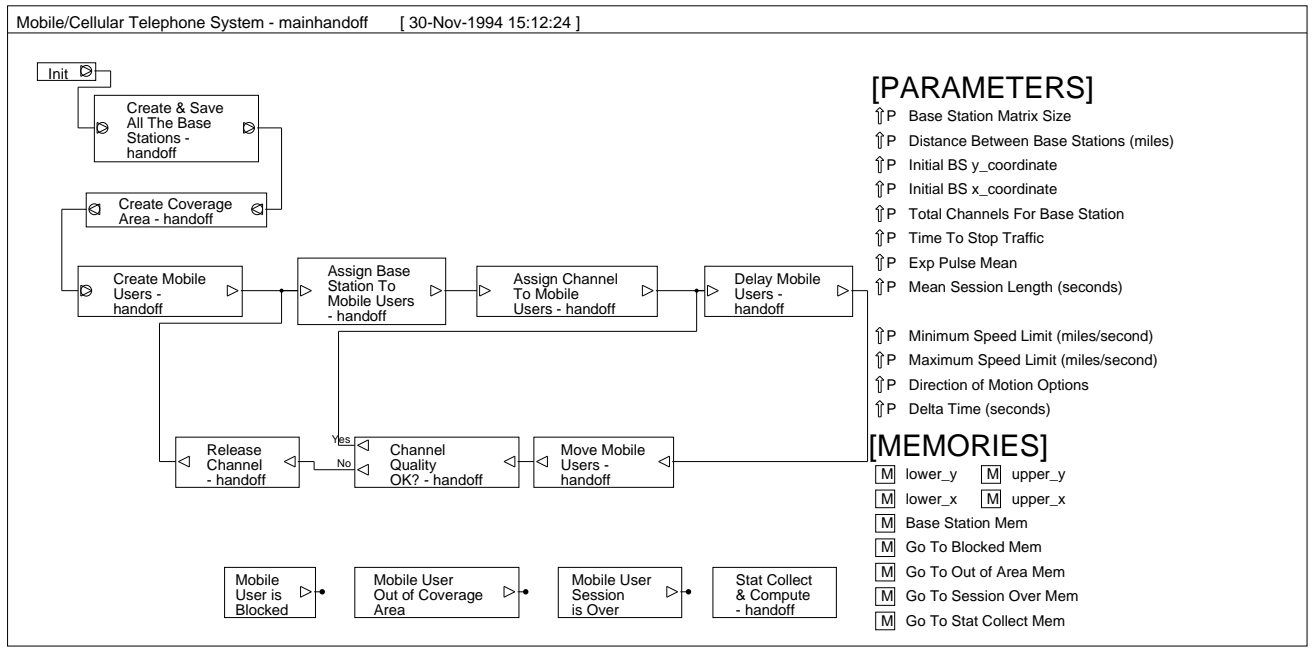

Figure 6: Top Level Mobile System.

and they are located in a square array. They all have the same number of channels, Total Channels for Base Station. Each cell area can be approximated as a circle with radius:

Distance Between Base Stations

Mobile hosts are created in Create Mobile Users. All the mobile parameters are uniformly distributed, except the session duration which is exponential and agrees with equation 1 of our analysis. New mobiles enter the system with an interarrival time of Exp Pulse Mean. The mobile host will arrive at a location uniformly distributed anywhere in the area covered by all cells. Since a mobile makes one call in its lifetime, a mobile host represents a single connection. Thus,

$$
\lambda_{R}=\frac{1}{(\text { Exp Pulse Mean })(\text { Total Cell Area })(B)}
$$

where $B$ is the number of base stations.

The following modules act upon the mobile hosts throughout their lifetime. The mobile hosts are assigned the nearest base station Assign Base Station to Mobiles Users, and an available channel from that base station Assign Channel to Mobile Users. Then all mobile hosts dwell in their cell areas for time Delta Time Delay Mobile Users. The mobile host then moves to its next location which may be uniformly chosen from Direction of Motion Options and may lead to a new cell or completely outside the cellular system Move Mobile Users.

After moving, the quality of signal is checked and if below a given criteria ${ }^{2}$ the channel is released (Release Channel) and the mobile host is reassigned to a new base station (Assign Base Station to Mobile Users).

\footnotetext{
${ }^{2}$ In this case, if the distance between a mobile host and its currently assigned base station is greater than $\frac{2}{3} \sqrt{D^{2}-\left(\frac{D}{2}\right)^{2}}$ where $D$ is the distance between base stations, then the channel quality is considered unacceptable.
} 
Note that the direction of travel by a mobile user is uniformly chosen from North, South, East, or West. A more sophisticated analysis of speed and direction is contained in [6].

The simulation results are shown in Figures 7 and 8. These results agree with the results of the analysis shown in Figures 4 and 5.

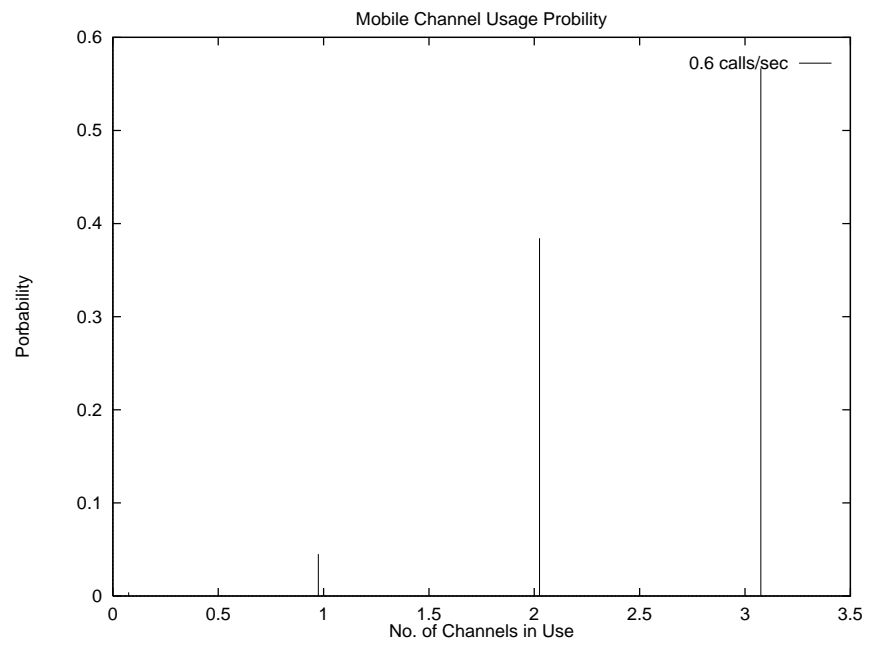

Figure 7: Channel Usage Probability for 0.6 Call/Sec.

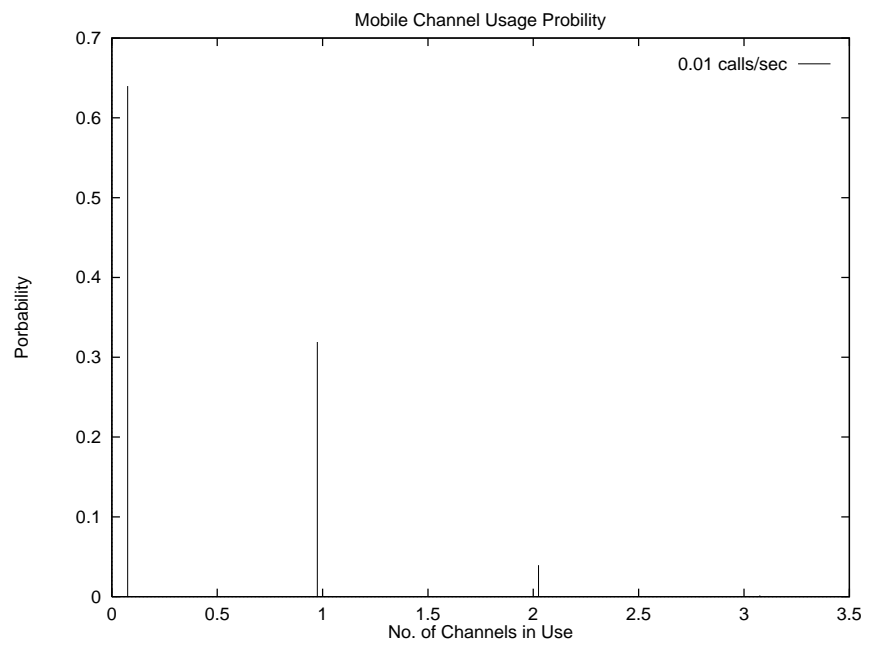

Figure 8: Channel Usage Probability for 0.01 Call/Sec. 


\section{Summary}

This paper attempted to extend [1] to a mobile environment. It also presented the results of a simulation of a mobile environment in preparation for simulating the extension. The mobile environment adds several new dimensions to fixed communications analysis, such as cell area, speed, direction, channel holding time, channels used at a base station, frequency of handoffs. This simulation concentrated on finding channel hold time, $T_{H}$, and the average number of channels used, $P_{j}$, which are required for the extension of [1].

This paper also suggested areas for further research such as extending [1] to mobiles which are treated as multiple CBR sources, analyzing queued handoffs, and enhancing the channel hold time and number of channels used by using a PDE for the speed and direction analysis as in [6].

\section{References}

[1] D. Anick, D. Mitra, and M. M. Sondhi. Stochastic theory of a data-handling system with multiple sources. BSTJ, 61:1871-1894, 1982.

[2] Stephen F. Bush, Sunil Jagannath, Joseph B. Evans, and Victor Frost. A Control and Management Network for Wireless ATM Systems. In Proceedings of the International Communications Conference '96, pages 459,463, June 1996. URL: http://www-ee.uta.edu/organizations/commsoc/commsoft.html.

[3] Stephen F. Bush, Sunil Jagannath, Ricardo Sanchez, Joseph B. Evans, Victor Frost, and K. Sam Shanmugan. Rapidly Deployable Radio Networks (RDRN) Network Architecture. Technical Report 10920-09, Telecommunications \& Information Sciences Laboratory, July 1995. URL: http://www.tisl.ukans.edu/ sbush .

[4] Daehyoung Hong and Stephen S. Rappaport. Traffic Model and Performance Analysis for Cellular Mobile Radio Telephone Systems with Prioritized and Non prioritized Handoff Procedures. IEEE Transactions on Vehicular Technology, Aug 1986.

[5] William Y. Lee. Overview of Cellular CDMA. IEEE Transactions on Vehicular Technology, May 1991.

[6] Kin K. Leung, William A. Massey, and Ward Whitt. Traffic Models for Wireless Communications Networks. IEEE Journal of Selected Areas in Communication, October 1994. 